\title{
Experiences of weight-related stigma among low-income rural women of higher weights from the midwestern United States
}

\author{
Katherine Hughes, ${ }^{1}$ Andrea E. Bombak, ${ }^{2}$ Samuel Ankomah ${ }^{1}$ \\ ${ }^{1}$ School of Health Sciences, Central Michigan University, Mount Pleasant, MI, USA; ${ }^{2}$ Department of Sociology, University of New \\ Brunswick, NB, Canada
}

\begin{abstract}
Weight-related stigma and stereotypes are widespread. Despite established research highlighting the disproportionate disparities rural individuals face, studies focusing on women in rural and low-income environments are underrepresented in the literature. The current study addressed these gaps in the literature using 25 in-depth interviews, which were analyzed using interpretive phenomenological procedures. The research questions were: i) what are low income rural women of higher weights' understandings and experiences of weight stigma in healthcare? and ii) to what extent do their experiences of stigma affect or do not affect their healthcare-related behaviors? Sixteen women in the study experienced weight-related stigma in healthcare. Many delayed their care while others felt their care was essentially denied. Findings indicate that more can be done to address weight-related stigma.
\end{abstract}

\section{Introduction}

\section{Weight-related stigma}

The public perception of individuals of higher weights is affected by media that propagate weight stigma. ${ }^{1}$ Weight discrimination in society often goes unchecked, and people of higher weight are subject to social blame based on com-

Correspondence: Katherine Hughes, School of Health Sciences, Central Michigan University, Mount Pleasant, MI 48859, USA.

Tel.: +1.517.902.6262.

E-mail: hughe1k1@cmich.edu

Acknowledgments: the authors thank participants and community partnerships for recruitment opportunities.

Key words: Weight; Stigma; Low-income; Rural; Women.

Contributions: $\mathrm{KH}, \mathrm{AB}$ completed research interviews; $\mathrm{KH}$, SA data coding, worked with $\mathrm{AB}$ on data analysis. $\mathrm{KH}$ manuscript completion in consultation with $\mathrm{AB}$.

Conflict of interest: the authors declare no potential conflict of interest.

Funding: the work was supported by a College of Health Professions Early Career Grant, Central Michigan University.

Received for publication: 17 September 2018.

Revision received: 18 February 2019.

Accepted for publication: 18 February 2019.

This work is licensed under a Creative Commons Attribution NonCommercial 4.0 License (CC BY-NC 4.0).

CCopyright K. Hughes et al., 2019

Licensee PAGEPress, Italy

Qualitative Research in Medicine \& Healthcare 2019; 3:25-31

doi:10.4081/qrmh.2019.7832 mon assumptions of laziness, overeating and oversimplifications of health status. ${ }^{1,2}$ The relationship between weight and health is often oversimplified and sensationalized. ${ }^{3}$ Rural, low-income women of higher weights occupy multiple disadvantaged social locations that may influence their health. ${ }^{4}$ As these women may already be marginalized by sexism or classism, the added burden of weight-related stigma may exacerbate ongoing health issues and produce a multiply disadvantaged social status. ${ }^{5}$ Individuals experience multiple, intersecting social locations at any one time that may accord them different disadvantages and advantages in particular contexts. In one of only few studies that examined weight from this intersectional perspective, Himmelstein et al. found that low income individuals experience more weight-related stigmatization. ${ }^{5}$ Rural residents also must cope with added stereotyping of backwardness and unsophistication. ${ }^{6}$ Despite established research highlighting the disproportionate disparities rural individuals face, ${ }^{7}$ women and weight-related stigma in rural and lowincome environments are not studied often, in part, perhaps because these women are perceived as being less concerned with their bodies. ${ }^{8}$

Women experience harsher weight stigma in a variety of settings, and weight stigma in women is experienced at a lower weight compared to men. ${ }^{1}$ Evidence of stigma and its impact on psychological and behavioral responses should be viewed as a vital determinant of health that greatly influences health and health inequalities. ${ }^{9}$

\section{Weight-related stigma and healthcare}

Weight-related stigma and stereotypes are widespread across society, including among healthcare professionals. ${ }^{2,10}$ Mounting evidence indicates that weight-related stereotypes negatively influence the quality of care higher weight individuals receive, worsening disparities and barriers to 
care ${ }^{2}$ Consequences of weight stigma include avoidance of medical care, poorer quality of care, medication non-adherence, disordered eating, reduced weight loss, inactivity, psychosocial stress, provider distrust, and poorer mental health. ${ }^{2,10}$ In their review on the effects of weight stigma on health, Phelan et al. ${ }^{2}$ reviewed numerous studies that indicate that those who experience weight-related stereotyping in healthcare settings delay care and avoid providers due to the stress caused by their negative treatment.

In a review of reports on weight-related stigma, Flint ${ }^{11}$ suggests that the breakdown of the patient-practitioner relationship, which can involve the use of alarmist terms to describe excess fatness, has contributed to patients avoiding and even cancelling appointments. In a study that helps elucidate why women of higher weights are less likely to seek care than thinner counterparts, Mensinger et al. ${ }^{12}$ found that women of higher weight experience internalized weight-related stigma that contributes to body guilt and shame. This guilt and shame leads to increased healthcare-related stress causing healthcare avoidance. The evidence that individuals of higher weight may fail to report health concerns and delay/avoid needed healthcare emphasizes the paramount importance of understanding the experiences of stigma in those disproportionately affected by additional barriers and disadvantages ${ }^{4}$ which has not been a focus of weight-stigma research. Despite the potentially highly salient effect of weight-related stigma, we know little about these factors in low-income and rural locales whose residents may experience compounding disparities and barriers to receiving healthcare.

The inadvertent or deliberate stigmatization of women of higher weights and the barriers it creates should be explored in order to better understand how to promote health at every size. The current exploratory study helps to elucidate the role of weight stigma in terms of healthcare behaviors and beliefs of rural women of higher weights.

\section{Research objective}

In the current study, we seek to address the abovementioned gaps in the literature using 25 in-depth interviews, conducted in the rural Midwest of the United States of America, and analyzed using interpretive phenomenological analysis. We address the following questions: i) what are low income rural women of higher weights' understandings and experiences of weight stigma in healthcare? and ii) to what extent do their experiences of stigma affect or do not affect their healthcare-related behaviors?

\section{Materials and Methods}

\section{Study design}

This study employed in-depth interviewing and Interpretive Phenomenological Analysis (IPA) practices. IPA equips researchers with the skills necessary to understand the experiences of individuals by capturing their narra- tives and providing transparent, comprehensive, and organized interpretations or sense making. ${ }^{13,14}$ Just as individuals are conducting sense making of their own lived experiences, IPA requires the researcher to make sense of the participant's experience. This two-way relationship is the process of a double hermeneutic, enabling interpretations to be made by the researcher based on participants' narratives related to the phenomenon of interest. Analysis in IPA is based on ideography; the event of interest in each individual case is identified and all interviews are examined to identify patterns, themes, and divergence across all individuals. ${ }^{13,14}$ IPA is not a prescriptive approach - it provides freedom and flexibility, but it also requires crosschecking at multiple points during data collection and analysis. ${ }^{15}$ Given that IPA focuses on participants' lived experience and sensemaking practices, it provides an appropriate means to understand how individuals make sense of their weight stigma experiences. ${ }^{16}$

\section{Participant selection}

We recruited individuals from flyers, listservs, and snowballing at community groups and sites serving lowincome families (food pantries, clinics, college campus, and community centers). The inclusion criteria for this study included self-identifying as female, currently or have ever been considered obese (Body Mass Index $\geq 30$ ), less than twice the poverty guidelines set forth by the Department of Health and Human Service or enrolled in an income-based social assistance program, and living in a rural Michigan area defined by the US Census Bureau urban-rural classification system. We excluded individuals from the study if they were non-English speaking. Individuals interested in participating were contacted via email or phone to schedule an interview. We recruited twenty-five participants, which is considered the upper limit for phenomenological studies ${ }^{17}$ Participants were given $\$ 50$ to reimburse their time and travel.

\section{Setting}

Data collection was conducted at public places of the participants' choosing. The majority of interviews took place at McDonalds fast food locations, as that is one of few public meeting places in rural areas that is accessible and convenient. A total of 25 women (age: 22-90, $M=40.5$, $S D=19.87)$ participated. Participants self-identified as White (83.33\%), Black (8.33\%), Latin American (4.17\%), and American Indian (4.17\%). Most of the participants had completed secondary school $(20.83 \%)$ or some university $(20.83 \%)$. Most of the participants were not working in the labor force (a caregiver who does not work by preference/on leave/unemployed) $(45.83 \%)$ or were retired (25\%).

\section{Data collection}

Data was collected during face-to-face interviews, using semi-structured interview guides (Table 1). Smith 
and Osborn suggest the utilization of semi-structured interviews in IPA to allow flexibility in obtaining participants' unique stories and for rapport building. ${ }^{16} \mathrm{We}$ audio-recorded interviews, which were then transcribed by a third party. We recorded fieldnotes after each interview to depict the interactions during the interview, to elaborate on emotions expressed, and to capture visual or verbal cues that were not captured by the recording. ${ }^{18}$ On average, interviews lasted 46 minutes, ranging from 2084 minutes (Median: 42 minutes, Mode: 60 minutes). $\mathrm{KH}$ was the interviewer and was trained by $\mathrm{AB}$, who has extensive experience conducting qualitative interviews. $\mathrm{KH}$ had previous contact with some participants through recruitment at a community food distribution site.

\section{Data analysis}

Prior to the first interview, $\mathrm{AB}$ deductively began developing the initial code list by mapping the field $d^{19}$ and considering relevant prior studies and theory that may prove pertinent to the present study. The first interviews then informed the code list; new fields were added from relevant themes arising in the interviews. All three authors were responsible for coding the interviews using NVivo 11 software; coders met weekly to discuss patterns and allow for investigator triangulation. Coding was done using a stepwise approach, informed by IPA procedures. ${ }^{16}$ Each line of the interviews was read and re-read to identify words or phrases that match with the field; text that converged and diverged from the existing code were included. Each interview was summarized by the interview's initial coder and a subsequent coder. Separate codes were then combined to identify patterns with similar meaning; these patterns in meaning were identified as emergent themes. We then looked at all across interviews using coding tables as a way of detecting patterns among participants. We used these coding tables to reaffirm and refine the themes that emerged and to detect patterns within emergent themes.

\section{Results}

Below, we examine the two main themes we identified by using quotations from our interviews with participants.
Their experiences with weight-related stigma will be described first and then, we will move to analyze how participants were affected by weight-related stigma in a healthcare setting. The subheadings represent sub-themes within each of the three main themes; quotes that support each category and exceptional disconfirming quotes will be included.

\section{Weight-centrism in healthcare}

Sixteen participants described feelings of judgment or assumptions by healthcare professionals (mostly physicians) that were solely related to weight. Most participants felt physicians made judgments about their health without taking into consideration context, background, or their personal history. One participant mentioned that she believes physicians are unequipped and ill trained to work with patients of higher weights. This notion was shared by participants who describe nurses speaking about patients of higher weights in a demeaning way and physicians who give recommendations that the women in this study felt were unachievable given the presence of a conflicting injury or illness. The sense that physicians reduce complicated lifeways is described by Naomi:

Dealing with medicine...the doctor looks at you and already they can tell that they've analyzed you ... by seeing...one thing and that's not always the case.

The situation that Naomi described was a very common occurrence for the women in this study. For example, Taylor shared a similar experience of assumptions based only on weight by healthcare professionals. Taylor who has chronic health issues and sports-related injuries describes her experiences in healthcare:

I have a really [bad] knee from playing softball in high school, and they automatically say that it's just because of your weight. Or...I have a pinched nerve in my back from work, and it's like, "Well, you need to lose weight." Which, I've done... and those problems don't go away with losing weight ...they're a chronic issue...they just automatically assume everything wrong with you is because of your weight.

Participants also described hearing statements made by physicians that led them to believe they were being

Table 1. Sample of semi-structured interview guide.

\section{Question 1}

How do you think society treats women of higher weights?

In what ways do you think society has or has not treated you differently due to your size?

\section{Question 2}

How do you think healthcare providers treat women of higher weights?

How do you think those experiences are related (or not related) to your size?

\section{Question 3}

How do you think those experiences of being judged (or not judged) for size has affected women's health? Lifestyle? Willingness to go to the doctor? How do you think experiences of being judged (or not judged) for size has affected your health? Lifestyle? Willingness to see healthcare providers? 
judged because of their weight. Statements made by physicians to the participants concerning their weight followed the same general pattern. For example, Contessa shared a statement made to her by a physician that encompasses what many of the participants expressed:

[We] wouldn't have to be seeing you for this issue if you'd lose some weight...we wouldn't have to do this test, ya know. Wouldn't cost as much for your medical care if you could just lose some pounds.

Weight-centrism present in the physician-patient relationship was described by many women. While variations in its manifestation were present, it can be summarized as physicians indiscriminately attributing all their health conditions or illnesses only to their current weight. Weight-centrism may have seemed especially egregious for women in this study who were living in relatively isolated locales on limited budgets. These individuals were already coping with barriers to healthfulness and class-based stereotypes. As described by Lucy:

I'm retired and older and having to live on a fixed income.... if you use a Bridge Card people [act] like it's coming out of their pocket...I can't afford to buy food, but the government's helping me pay for it so people think, well, I pay taxes so into government programs, you know, so it's coming out of my pocket. Why are you so fat and why are you so lazy...

Therefore, in addition to reinforcing simplistic conflations of health and weight status, weight-centrism dismissed the health-damaging inequities participants encountered in their everyday lives.

\section{Effects of weight-related stigma}

Every participant mentioned the effects of weight-related stigma that they had experienced. Most expressed that the situation elicited an emotional response (e.g. cut down, depressed, hopeless, confused, and angry); with respect to experiences in healthcare, some went further, describing consequent feelings of evasiveness toward seeking care (Avoidance of care), and/or feeling like they were denied care to which they were entitled (Denial of care).

\section{Avoidance of care}

Participants described a variety of situations that would cause them to avoid healthcare. For some participants, avoidance was due to the seeming futility of speaking to physicians. Naomi describes her reaction to the way she is treated in healthcare:

They're going to say, if you're overweight, "You're overweight, you need to lose weight"...it kind of hurts hearing that over and over and over again to the point where you get discouraged to go to the doctor anymore.

For participants like Naomi, a visit to the physician was unnecessary. The outcome of the visit was a foregone conclusion (being told to lose weight) and would simply produce emotional hurt.

Taylor similarly describes one of her experiences dur- ing pregnancy that led her to cease care unless she was very sick:

Then going to the $O B$ when you're heavy versus if you're not heavy, there's... a huge difference. They ... automatically assume you eat trash all day, or you're already gonna have gestational diabetes, or your baby's gonna be huge and they just wanna automatically schedule you for a C-section (laughs)...

Having their lives reduced to weight-based stereotypes and encountering persistent negativity concerning their size motivated some women to forgo medical treatment. Importantly, knowledge, which was used to counter-productively reinforce to Taylor the risks associated with her pregnancy, was viewed as insufficient preparation for physicians in treating those with higher weight. As emphasized by Mia in explaining why she delays healthcare treatment:

I think healthcare professionals get to a point where they see their daily routine as a job...if the healthcare workers aren't overweight, they still have the same attitudes that people on the street have. Just because you have a degree in medicine doesn't make you any nicer.

While some participants delayed or avoided care because they expected to encounter weight-centric treatment, others described more material reasons for avoiding care. Bette, who has had both positive and negative experiences with physicians, explains what makes her unwilling to go see a physician:

Mainly just the...cost...I do have Medicare...but the copays are still high....Medicare is like over a hundreddollars a month...that they take out of my...disability...

Thus, participants experienced multiple barriers to seeking respectful, effective healthcare. For some, given the likelihood of encountering reductionist commentary on their size, the outcome of compounding material and social inequities was to avoid healthcare.

\section{Denial of care}

Other participants described what can be interpreted as a form of denial of care. This manifested most often because of weightcentrist ideals held by physicians preventing any course of action being taken during the appointment. Participants were persistently told to lose weight, eat healthy, and exercise leaving them feeling like the condition or illness for which they sought medical care for was not being addressed. For example, Jen (whose husband had only one episode of high blood sugar and eats a healthy diet) summarized her frustration with healthcare professionals:

They just like, "Oh, you have type 2 diabetes." It's just their go-to. They don't wanna look...intensively... They don't wanna look into what's actually causing that.

Because of the frequent elision of higher weight status with diabetes, Jen's husband was essentially denied care for his true issue. Rogue had a similar experience with a physician saying:

I went to see a doctor about potentially rheumatoid 
arthritis...this gentleman...never saw me before, and he saw me for less than 10 minutes, I timed it. And he had just looked at my hands and said, "This isn't inflammation, it's just because you're overweight." and walked out the door ...I think it's easier for health professionals to call me out on not exercising as much, or that's where they go straight to. I mean they're not entirely wrong, but I wonder if it's just because of the way I look that they, they go quickly to that.

Rogue, again, felt that her actual concern (rheumatoid arthritis) were easy to dismiss for physicians because of assumptions linked to higher weight status.

Some participants recounted experiences of family members and friends that influenced their own healthseeking behavior. Abbie describes the experience her father had with healthcare professionals in trying to address high blood sugar levels. The problem persisted despite her father following medical advice:

My father just died...He said, "I will not go to the healthcare system because...they told him the same thing, Lose weight. Your sugar's not gonna come down until you lose weight." Well, he lost weight, sugar was still acting up...It kind of has affected me as far as like healthcare because it makes me not even want to go to the doctor...I just want to be healthier, you know, but you're not giving me advice really, you're just telling me what I already know.

Abbie mirrors what many of the participants expressed, seeking care becomes a chore where many felt they needed to defend themselves, speak up, or fight to be believed when wanting to address their concerns pertaining to health and their bodies. Even when they advocated for themselves during appointments they felt their summons for help went unanswered. As Tamara states:

They treat me like I'm no good, you know... To set you aside and just blow you off instead of helping when you ask for help.

Thus, when participants did seek out care (which might involve considerable effort and resources), they were likely to encounter reductionist solutions based on assumptions concerning the link between health and weight. This denial of the care they actually sought could produce an unwillingness to engage further with healthcare providers.

\section{Discussion}

Low income, rural women of higher weight experience weight-related stigma. Research suggests that stigma may affect quality of care and health-seeking behaviors. The current study adds to the body of literature regarding experiences of weight-related stigma, providing insights from low-income rural women of higher weights who are sometimes perceived as indifferent to societal body standards. ${ }^{8}$ While there are few studies that examine stigma, well-being, and health-seeking behaviors among low-income and rural women, each theme can be compared with similar studies. Women who reported experiencing stigma in Himmelstein et al.' ${ }^{4}$ survey had higher BMI's and a lower average income than those who did not report experiencing stigma. Most participants mentioned stigmatizing interactions with their physicians. Weight-related stereotypes held by physicians have been abundantly measured and affirmed. Comparable to the studies reviewed by Phelan et al., ${ }^{2}$ participants in this study described feeling like they received lower quality care due to their weight. A previous study done by Bombak et al..$^{20}$ found that women classified as overweight or obese felt their reproductive care needs were denied based on their weight. The women in the current study described similar refusal of needed care by healthcare professionals who held weight-centrist ideals. Importantly, some participants stated they had neutral or positive interactions with healthcare professionals. All three participants who relayed positive experiences (characterized as timely and attentive) had the same family physicians since childhood.

Participants in the present study described similar reasoning leading to avoiding or delaying healthcare as identified by other researchers. These reasons included: not having a good rapport with healthcare staff and not receiving treatment on the basis of weight, ${ }^{11}$ a lack of communication and hurtful words to describe body fat, ${ }^{21}$ a perceived lack of resources, and prior negative experiences. $^{22}$ The avoidance of care for women of higher weights due to stigmatizing experiences has been established; however, how these encounter constitute a form of implicit (or explicit) denial of care through the dismissal of patients' concerns remains under-explored.

This research adds to the literature tasked with understanding stigma of underrepresented groups, but it is critical that more research is conducted that will aid in countering the deeply held assumptions regarding individuals of higher weights. More research must also be conducted that considers intersectional forms of marginalization. ${ }^{4}$ Participants in the present study were also coping with material and social disadvantage produced through living on lower incomes and in rural locations. Discrimination based on socioeconomic status is present in healthcare contexts. ${ }^{23}$ Rural spaces and lifestyles are depicted as backward and unsophisticated $^{6,24}$ and class-based assumptions tend to dismiss material barriers to well-being as forms of ignorance. ${ }^{25}$ More research will not only guide more respectful medical messaging, but it may help develop messaging that improves patients' self-image.

\section{Strengths and limitations}

Our findings help to articulate the experiences of those who frequently experience stigma and disadvantage and can be used to guide medical interactions and messaging that is more respectful for women at every size. For example, findings highlight potential disparaging messaging in healthcare and other sectors and should act as a catalyst to change the discourse surrounding the care of women 
of higher weights. The avoidance and denial of care subthemes can help inform dialogue related to perceived care for low-income rural women of higher weights.

To enhance rigor, we created audit trails in the form of NVivo memos and employed investigator triangulation throughout analysis. ${ }^{26-28}$ Professional transcription and reflexive fieldnotes, including a final reflexive summary consulted after the interview, added to the rigor of the study. ${ }^{26,27}$

Due to the nature of this study, the focus was on women, but the experiences of rural and low-income men would further add to the body of literature dealing with weight-related stigma. The sample was highly educated and mostly White. Studies on weight bias in general should aim for greater diversity and considerations of intersectionality. ${ }^{4}$ The small scale, qualitative nature of the study prohibits generalization, but similar themes have been identified elsewhere, suggesting transferability of findings. $\mathrm{KH}$, the main interviewer, is a slim female from a different class background, which may have affected rapport with participants.

\section{Conclusions}

In this interpretive phenomenological exploration of low income, rural women's experiences with weight stigma, all participants described experiencing some form of weight-related stigma. Many had experiences that fell into all the categories explicated in the results section: weight-centric ideals from healthcare professionals, and more than half revealed that the stigmatization from healthcare professionals produced evasion from seeking care or feeling like they were denied care. Weight-centrism during previous medical encounters contributed to the women in this study deciding that it was futile for someone with their body to seek care. As some of the women did not seek care until they were very ill or never sought care at all, this indicates weight stigma is a critical concern. Action needs to be taken across sectors to reduce barriers to medical care for rural residents, low-income individuals, and women of higher weights. A weight-neutral approach should be adopted for health communication. ${ }^{29}$ This approach maintains a holistic approach to health; an endorsement of sound research and prevention; and advocates for improved access to healthcare, resources for health promotion, social justice and stigma reduction. ${ }^{29}$ Such an approach would help alleviate the intersecting forms of disadvantages that kept participants marginalized within the healthcare system. Given the effects of weight-centrism on participants' health-seeking behaviors, public health policy and practice should promote non-stigmatizing messages that seek to promote healthy behavior without mention of body weight or size ${ }^{30}$ and that resist the common, damaging stereotyping of higher weight persons as ignorant, lazy, and over-indulgent,, 325 particularly among low-income, rural residents coping with similar classist assumptions. ${ }^{6,24}$ The findings from the current study point to the urgency of making public health messages more respectful by keeping weight out of health-related communications and pushing to eliminate intersections stigmas in every interaction and touch-point with the public.

\section{References}

1. Puhl R, Heuer C. The stigma of obesity: A review and update. Obesity 2009;17:941-64.

2. Phelan S, Burgess D, Yeazel M, et al. Impact of weight bias and stigma on quality of care and outcomes for patients with obesity. Obes Rev 2015;16:319-26.

3. Gard M, Wright J. The obesity epidemic: science, morality and ideology. Abingdon, Oxon: Routledge; 2005.

4. Himmelstein M, Puhl R, Quinn D. Intersectionality: An understudied framework for addressing weight stigma. Am J Prev Med 2017;53:421-31.

5. Grollman E. Multiple disadvantaged statuses and health: The role of multiple forms of discrimination. J Health Soc Behav 2014;55:3-19.

6. Beagan B, Chapman G, Johnston J, et al. Acquired tastes: Why families eat the way they do. Vancouver: University of British Columbia Press; 2015.

7. Meit M, Knudson A, Gilbert T, et al. The 2014 update of the rural-urban chartbook. 2014. Available from: https://ruralhealth.und.edu/projects/health-reform-policy-research-center/pdf/2014-rural-urban-chartbook-update.pdf

8. Bove CF, Olson CM. Obesity in low-income rural women: Qualitative insights about physical activity and eating patterns. Women Health 2006;44:57-78.

9. Hatzenbuehler M, Phelan J, Link B. Stigma as a fundamental cause of population health inequalities. Am J Public Health 2013;103:813-21.

10. Himmelstein M, Incollingo Belsky A, Tomiyama A. The weight of stigma: Cortisol reactivity to manipulated weight stigma. Obesity 2015;23:368-74.

11. Flint SW. Obesity stigma: Prevalence and impact in healthcare. Br J Obes 2015;1:14-8.

12. Mensinger J, Tylka T, Calamari M. Mechanisms underlying weight status and healthcare avoidance in women: A study of weight stigma, body-related shame and guilt, and healthcare stress. Body Image 2018;25:139-47.

13. Smith JA. Beyond the divide between cognition and discourse: Using interpretive phenomenological analysis in health psychology. Psychol Health 1996;11:261-71.

14. Smith JA. Evaluation the contribution of interpretive phenomenological analysis. Health Psychol Rev 2011;5:9-27.

15. Smith JA, Flowers P, Larkin M. Interpretative phenomenological analysis : theory, method and research. London: SAGE Publications Ltd; 2009.

16. Smith JA, Osborn M. Interpretative phenomenological analysis. In: Smith JA, ed. Qualitative psychology: A practical guide to research method. London: Sage; 2008. pp 53-80.

17. Creswell JW. Qualitative inquiry and research design: Choosing among five approaches. 2nd ed. Thousand Oaks, CA: Sage Publications; 2007.

18. Emerson RM, Fretz RI, Shaw LL. Writing ethnographic fieldnotes. Chicago: University of Chicago Press; 2003.

19. Wright J. Poststructuralist methodologies - The body, schooling and health. In: Evans J, Davies B, Wright J, eds. 
Body knowledge and control. Studies in the sociology of physical education and health. London, New York: Routledge; 2003. pp 34-59.

20. Bombak A, McPhail D, Ward P. Reproducing stigma: Interpreting "overweight" and "obese" women's experiences of weight-based discrimination in reproductive healthcare. Soc Sci Med 2016;166:94-101.

21. Dutton G, Tan F, Perri M, et al. What words should we use when discussing excess weight? J Am Board Fam Med 2010;23:606-13.

22. Brown I, Thompson J, Tod A, Jones G. Primary care support for tackling obesity: A qualitative study of the perceptions of obese patients. Br J Gen Pract 2006;56:666-72.

23. Zilioli S, Imami L, Ong AD, et al. Discrimination and anger control as pathways linking socioeconomic disadvantage to allostatic load in midlife. J Psychosom Res 2017;103:83-90.

24. McPhail D, Chapman GE, Beagan BL. The rural and the rotund? A critical interpretation of food deserts and rural adolescent obesity in the Canadian context. Health Place 2013;22:132-9.
25. Farrell LC, Warin MJ, Moore VM, Street JM. Socio-economic divergence in public opinions about preventive obesity regulations: Is the purpose to 'make some things cheaper, more affordable' or to 'help them get over their own ignorance'? Soc Sci Med 2016;154:1-8.

26. Attride-Stirling J. Thematic networks: An analytic tool for qualitative research. Qual Res 2001;1:385-405.

27. Creswell JW. Research Design: qualitative, quantitative, and mixed methods approaches. $4^{\text {th }}$ ed. Thousand Oaks, CA: Sage Publications; 2009.

28. Kaczynski J, Salmona M, Smith T. Qualitative research in finance. Aust J Manag 2013;39:127-35.

29. Tylka T, Annunziato R, Burgard D, et al. The weight-inclusive versus weight-normative approach to health: Evaluating the evidence for prioritizing well-being over weight loss. J Obes 2014;2014:1-18.

30. Puhl R, Peterson J, Luedicke J. Fighting obesity or obese persons? Public perceptions of obesity-related health messages. Int J Obes 2012;37:774-82. 